\title{
Vaccination against chlamydial and mycobacterial infections
}

Ph.D. Thesis

Ágnes Míra Szabó

Department of Medical Microbiology and Immunobiology

Faculty of Medicine

University of Szeged

Szeged, Hungary

2013 


\section{Introduction}

Metabolism and pathogenicity of intracellular bacteria (mycobacteria, Chlamydophila pneumoniae) were studied in the laboratory when I started my Ph.D. studentship. Possible vaccine targets were identified, RNA metabolism, glyoxylate cycle, regulatory proteins were investigated. My work focuses on recombinant BCG as an improved vaccine candidate, $\mathrm{ABC}$ transporter ATPase of $C$. pneumoniae and Mycobacterium tuberculosis, and low calcium response protein $\mathbf{E}$ as possible components of vaccines. There is a big competition in finding new drugs and vaccines among biotechnology and pharmaceutical companies and there is a vast difference between our budget and theirs. The investigation of intracellular bacteria is a very important research area. The survival and spread of these bacteria depend on their ability to replicate inside the eukaryotic cell. The similar life habit might be realized through the homologous proteins.

More efficient vaccination against C. pneumoniae and M. tuberculosis would be a great step in the prevention and treatment of these infections. For possible vaccine candidates we cloned LcrE protein encoded by $l c r E$ of type III secretion system (TTSS) from C. pneumoniae, as well as chlamydial ABC transporter ATPase protein. The genes for these vaccine candidate proteins were cloned into Escherichia coli expression vector and E. coli was transformed with them.

The survival of $M$. tuberculosis in infected macrophages requires the activity of isocitrate lyase (Icl), a key enzyme in the glyoxylate cycle. $i c l$ gene was cloned into $E$. coli and $E$. coli-mycobacterium shuttle plasmids and E. coli and Mycobacterium bovis BCG were transformed with them.

BALB/c and ApoB100-only LDLR-/- (B6;129S-ApoBtm2SgyLdlrtm1Her/J) mice were immunized either with the purified proteins or with recombinant M. bovis BCG bacterium and a eukaryotic expression vector.

\subsection{Vaccination against intracellular bacteria}

Vaccination against human pathogens is one of the best ways to put an end to the serious effects of certain diseases. In clinical use, vaccines consist of either killed or attenuated microbes such as influenza, polio, measles, tuberculosis or subunits of the organisms (Haemophilus influenzae type B, Meningococcus A and C, Hepatitis B). Long-lived immune responses (cell-mediated response and production of protective antibodies) are induced by this type of vaccines. Most current vaccines are able to induce the immune response and effective for diseases mentioned above. In addition, cell-mediated immunity (mediated by T lymphocytes) is crucial in the immune protection of intracellular microbes. Antigen-presenting cells (macrophages or dentritic cells) produce IL-12, which is the inducer of the functional Th1 response and the protection mediator is the effector cytokine, IFN- $\gamma$. At the same time, for diseases such as AIDS, malaria and other infections caused by intracellular pathogens, effective vaccines are not available.

\subsubsection{BCG vaccine versus new vaccines for tuberculosis}

Although M. bovis BCG (Bacille Calmette-Guérin) is the only approved vaccine used for the prevention of TB in humans, the protective efficacy of the BCG vaccine varies widely in different parts of the world, reaching a maximum of $78 \%$. The vaccine contains live attenuated strain of M. bovis which was attenuated between 1908 and 1920 by 231 serial passages. During 13 years in vitro passage, the virulent strain was seen to be less virulent for animals. BCG vaccine was first used in humans against tuberculosis in 1921. Since that time, BCG is the most widely used vaccine all over the world, but its protective value as anti-TB vaccine is questionable. BCG can protect children against severe form of the disease, particularly meningitis, but it seems to be much less effective, or even ineffective against the most prevalent form, pulmonary tuberculosis in adults. To reduce the global burden of TB, new vaccination strategies are needed, not only in newborns, but also in adolescents and adults.

Most current efforts to improve the level of protective immunity provided by BCG include the development of recombinant BCG vaccines expressing different antigens. A number of vaccines, diagnostic technologies, and drugs are in clinical development. 
Mycobacterium smegmatis, first identified in 1884, is a rapidly growing saprophyte, it can propagate one generation every 1-3 h. It is non-pathogenic and commensal in humans, and it acts as a powerful cell immunity adjuvant. Unlike other mycobacterial species, such as $M$. tuberculosis, that survive in host cells by inhibiting phagosome maturation, M. smegmatis is rapidly destroyed by phagolysosomal proteases in the phagosomes of infected cells. M. smegmatis has a number of properties that can make it an effective vaccine vector. This fast-growing mycobacterium is unable to arrest phagolysosome maturation and cannot evade intracellular killing. Moreover, its rapid clearance by the host differs from that of the M. tuberculosis or even the vaccine strain M. bovis bacillus Calmette-Guérin. Furthermore, M. smegmatis has been used as a vaccine vector because it activates dendritic cells and induces CD8-mediated immune responses. Finally, killed M. smegmatis has been shown to provide the same adjuvant activity as M. tuberculosis during the induction of experimental autoimmune encephalomyelitis.

\subsection{Possible vaccine candidates}

\subsubsection{ABC transporter ATPase}

ABC transporter ATPase encoded by Rv0986 gene of M. tuberculosis H37Rv strain, is involved in cell adhesion and entry, and plays a part in the inhibition of phagosome-lysosome fusion. The expression of ATPase protein gene is up-regulated by at least 20 -fold in mycobacterium during infection. These are the reasons why it can be a suitable vaccine candidate. The proteins which are involved in bacterial cell invasion might be similar in different intracellular bacteria. C. pneumoniae has a homologous ATPase protein, encoded by $C p B 0255$ gene. The purpose of this study was the cloning, over-expression and purification of chlamydial $\mathrm{ABC}$ transporter ATPase protein; examination of the immunogenicity and the protective ability of subcutaneous recombinant protein vaccination with chlamydial $\mathrm{ABC}$ transporter ATPase against C. pneumoniae infection in mice.

\subsubsection{LcrE protein}

The protein encoded by $l c r E$ is homologous to Yersinia YopN, a surface protein thought to be a TTSS response regulator, which senses either a host cell contact in vivo or Ca2+ concentration in vitro. It is located at the outermost position in the TTSS structure. The fact that LcrE protein is exposed in the EB suggests that the TTSS apparatus may also be fully assembled in extracellular chlamydiae, possibly to be used in early events of cell infection, e.g. in order to assist the entry of chlamydiae into the host cell and the successful establishment of early chlamydial inclusion vacuole. Increasing expression of $\mathrm{LcrE}$ towards the end of the $C$.

pneumoniae infection cycle was detected. The fact that LcrE appears to be presented to antibodies on the surface of EB makes this protein a possible vaccine candidate. A protective effect of recombinant LcrE protein mixed with Freund's adjuvant given subcutaneously against $C$. pneumoniae challenge in a hamster model was described. The protective effect of immunization with LcrE protein mixed with Freund's or Alum adjuvant against experimental C. pneumoniae infection in mice was earlier described by our group. Studies have shown that cell-mediated immunity is necessary for protection against $C$. pneumoniae in mice. In immunization models DNA has been used to induce Th1 type immunity against $C$. pneumoniae. In several studies, a partial protection against the infection has been observed in mice vaccinated with naked DNA carrying genes for major outer membrane protein, $60 \mathrm{kDa}$ heat shock protein or $\mathrm{ADP} / \mathrm{ATP}$ translocase.

The purpose of our study was to test the immunogenicity and the protective ability of intramuscular $l c r E$-DNA immunization and $l c r E$-DNA priming/recombinant LcrE protein booster immunization regime against $C$. pneumoniae infection in mice.

\subsection{Aims}

The purpose of this study was:

- The over-expression and purification of chlamydial LcrE and ABC transporter ATPase proteins in large quantities

- To clone the genes for these vaccine candidate proteins into Escherichia coli expression vector and to transform E. coli with them 
- The effect of Icl:

$>\quad$ icl gene was cloned into E. coli and E. coli-mycobacterium shuttle plasmids and E. coli and Mycobacterium bovis BCG were transformed with them.

- Comparison the immunogenicity and the ability of protein vaccination, BALB/c and ApoB100-only LDLR-/- (B6;129S-ApoBtm2SgyLdlrtm1Her/J) mice were immunized either with the purified proteins or with recombinant $\mathrm{M}$. bovis BCG bacterium and a eukaryotic expression vector.

\section{Materials and Methods}

\subsection{Bacterial strains and growth conditions}

M. bovis BCG, E. coli $\mathrm{DH} 5 \alpha$, E. coli $\mathrm{HB} 101$ and C. pneumoniae CWL029 (ATCC)

strains were used. E. coli strains were routinely grown in LB (Luria-Bertani) medium supplemented with the appropriate antibiotic(s). M. bovis BCG was grown in Middlebrook $7 \mathrm{H} 9$ broth or 7H10 agar (Difco Laboratories, Detroit, MI, USA) enriched with 10\% Middlebrook OADC (oleic acid/albumin/dextrose/catalase) (Difco) and 0.05\% Tween 80 (Sigma-Aldrich Chemie GmbH, Steinheim, Germany) with the appropriate antibiotic. C. pneumoniae was propagated in HEp-2 cells.

\section{2 Plasmid constructions}

\subsubsection{LcrE plasmids}

A 1200 bp fragment containing the lcrE (GeneID: 895078; Locus tag: CPn0324) gene was amplified by PCR, using the following primers: E1 5'-GGA GGC ATA TGG CAG CAT CA-3' and E2 5'-CAC AGG ATC CGT ATT GGT TTT GCA TGG C-3' for ligation to prokaryotic expression vector (p6HisF-11d), the resulting plasmid was pLCRE. E3 5'-GCT AAG CTT ATG GCA GCA TCA GGA-3' and E4 5'-TTA GGG CCC GTA TTG GTT TTG CAT GGC-3' for ligation to eukaryotic expression vector ( $\mathrm{p} \Delta \mathrm{RC})$. C. pneumoniae CWL029 DNA was used as template. Primers were planned by using the sequence of CWL029.

PCR was performed in a GeneAmp II thermocycler (Applied Biosystems, Foster City, CA, USA) with Advantage GC cDNA polymerase (Clontech, Mountain View, CA, USA) and the amplification conditions were set as recommended by the manufacturer. The amplified DNA for eukaryotic expression vector ( $\mathrm{p} \Delta \mathrm{RC}$ ) was digested with HindIII and ApaI and inserted into the $\mathrm{p} \Delta \mathrm{RC}$ by digesting it with the same enzymes. The resulting plasmid was $\mathrm{p} \Delta \mathrm{RCLcrE}$. For DNA immunization we purified recombinant $\mathrm{p} \Delta \mathrm{RCLcrE}$ and $\mathrm{p} \Delta \mathrm{RC}$ plasmids in large scale by GenElute $^{\mathrm{TM}}$ HP Endotoxin-Free Plasmid Megaprep Kit from Sigma.

\subsubsection{ABC transporter ATPase plasmid}

A $681 \mathrm{bp}$ fragment containing the gene of ABC (ACP) (GeneID:33241335; Locus tag: $\mathrm{CpB} 0255)$ gene from $C$. pneumoniae was amplified by PCR, using the following primers: AB3 5'-GGG CAT ATG TCC TTA CTT ATA GAA-3' and AB4 5'-AAC GGA TCC AGA GGG AGT GTT TTC-3' and C. pneumoniae CWL029 DNA as template. Primers were planned by using the sequence of CWL029. This fragment was digested with NdeI and BamHI and inserted into $\mathrm{p} 6 \mathrm{HisF}-11 \mathrm{~d}(\mathrm{icl})$ by digesting with the same enzymes and replacing the $i c l$ gene. The resulted plasmid was pACP.

PCR was performed in a GeneAmp II (AppliedBiosystems, Foster City, CA, USA) thermocycler with Advantage GC cDNA polymerase (Clontech, Mountain View, CA, USA) and the amplification conditions were set as recommended by the manufacturer.

\subsection{3 pMV262(icl) plasmid}

We constructed pMV262(icl), which carries $i c l$ after the mycobacterial icl promoter (GenBank accession no. CAE55284.1) in pMV262. I1 5'-ACT ATC TAG ATC CGC AGG ACG TCG A-3' and I2 5'-GTA AGC TTC AGA CTA GTG GAA CTG G-3' primers were used to synthetize $i c l$ with $M$. tuberculosis $\mathrm{H} 37 \mathrm{Rv}$ chromosomal DNA as template. Primers were planned by using the sequence of H37Rv. The amplified DNA was cut with XbaI and HindIII and inserted into pMV262 opened with the same enzymes. 
PCR was performed in a GeneAmp II thermocycler (Applied Biosystem, Foster City, CA, USA) with Advantage GC cDNA polymerase (Clontech, Mountain View, CA, USA) and the amplification conditions were set as recommended by the manufacturer.

\subsection{Gene copy number determination}

The High Pure PCR Template Preparation Kit (Roche Diagnostic GmbH, Mannheim, Germany; Cat. No.: 1796828) was used for DNA Extraction. Each DNA sample was analysed in triplicate. The reaction volume was $15 \mu \mathrm{l}$, containing $3 \mu 1$ of DNA, $1 \mu \mathrm{mol} / 1$ of each of the primers $(2 \mu 1), 7.5 \mu 1$ of reaction buffer (IQ ${ }^{\mathrm{TM}} 2 \mathrm{X}$ Supermix, Bio-Rad Laboratories, Hercules, CA, USA) $0.6 \mu 1$ of EVAGreen (20x EVAGreen ${ }^{\mathrm{TM}}$ Biotium Inc., Hayward, CA, USA) and $1.9 \mu 1$ of distilled water. We used the forward primer 5'-AGC GCA TAT GTC TGT CGT CGG-3' and reverse primer 5'-GTA AGC TTC AGA CTA GTG GAA CTG G-3' for icl. The reference gene (GeneBank accession no. O53899) primer set was 5'-TGG CAT ATG AAC CGG CAA CCT ATC-3' and 5'-GAG GAT CCT CAT TCA TAG GAC GTG-3'. The primer sets have very similar efficiencies. A BIO-RAD CFX 96 instrument (Bio-Rad, Hercules, CA, USA) was used for quantitation. The PCR conditions were initial denaturation at $95^{\circ} \mathrm{C}$ for $10 \mathrm{~min}$, followed by 35 cycles of denaturation $\left(94{ }^{\circ} \mathrm{C}\right.$ for $\left.20 \mathrm{~s}\right)$, annealing $\left(55^{\circ} \mathrm{C}\right.$ for $\left.30 \mathrm{~s}\right)$ and extension $\left(68^{\circ} \mathrm{C}\right.$ for $\left.45 \mathrm{~s}\right)$. The emitted fluorescence was measured after the extension step.

Quantitation was performed by online monitoring for identification of the exact time point at which the logarithmic linear phase could be distinguished from the background (crossing point). The gene copy number was calculated by the $\Delta \mathrm{Ct}$ method with Bio-Rad CFX Manager 1.1 Gene Expression software.

\subsection{Protein analysis techniques}

\subsubsection{Expression of LerE and ACP}

For over-expression, E. coli HB101(pGP1-4) cells carrying either pLCRE or pACP plasmids were grown and treated according to the method of Tabor and Richardson. Briefly, cells containing the plasmids were grown at $32{ }^{\circ} \mathrm{C}$ in LB medium in the presence of the required antibiotics (ampicillin, kanamycin). Over-expression of proteins was induced by shifting the temperature to $42{ }^{\circ} \mathrm{C}$ for $20 \mathrm{~min}$. After induction, the temperature was shifted down to $37^{\circ} \mathrm{C}$ for an additional $30 \mathrm{~min}$ or longer, cells were harvested by centrifugation and cell pellets were frozen.

\subsubsection{Purification of LerE and ACP}

Cell lysates were prepared by resuspending the frozen cell pellets in lysis buffer ( 50 $\mathrm{mM}$ sodium phosphate, $300 \mathrm{mM}$ sodium chloride, $\mathrm{pH} 7,0)$ containing protease inhibitor cocktail (Sigma) and lysozime (Sigma) $(0,75 \mathrm{mg} / \mathrm{ml})$. Bacteria were opened by sonication. After centrifugation, either LcrE or ACP proteins were purified from the supernatant using TALON CellThru Resin (Clonetech), following the vendor's instructions.

\subsubsection{Protein detection}

Sodium dodecyl sulfate-polyacrylamide gel electrophoresis (SDS-PAGE) was performed according to Laemmli. Gels were stained with Coomassie brillant blue.

\subsubsection{Electroporation of $M$. bovis BCG}

Competent M. bovis BCG prepared in $10 \%$ glycerol was transformed with pMV262 or pMV262(icl) by electroporation with a Gene Pulser (Bio-Rad, München, Germany) set at $2.5 \mathrm{kV}$ and $25 \mu \mathrm{F}$, and with the pulse controller resistance set at 1,000 $\Omega$. Transformed BCG was selected on Middlebrook 7H10 agar plates supplemented with $30 \mu \mathrm{g} / \mathrm{ml}$ kanamycin.

\subsection{Immunological methods and toxicity testing}

\subsubsection{Immunization method of LcrE}

Specific-pathogen-free 13-week-old, female BALB/c mice were obtained from Charles River Laboratories UK (Kent, England). Mice were maintained under standard husbandry conditions at the animal facility of the Department of Medical Microbiology and Immunobiology, University of Szeged and were given food and water ad libitum. The mice in groups of 10 were immunized: (Group 1) with $50 \mu \mathrm{g} \mathrm{p} \Delta \mathrm{RC}$ plasmid intramuscularly once and 4 weeks later with the purified LcrE protein diluted in PBS at a dose of $20 \mu \mathrm{g}$ mixed with $25 \mu \mathrm{l}$ Alum (Aluminium hydroxide Gel, Sigma) in $150 \mu$ l subcutaneously into the tail base; or (Group 2) with $50 \mu \mathrm{g}$ 
$\mathrm{p} \triangle \mathrm{RCLcrE} \mathrm{plasmid} \mathrm{intramuscularly} \mathrm{two} \mathrm{times} \mathrm{with} \mathrm{4-week} \mathrm{interval,} \mathrm{or} \mathrm{(Group} \mathrm{3)} \mathrm{with} 50 \mu \mathrm{g}$ $\mathrm{p} \Delta \mathrm{RCLcrE}$ plasmid intramuscularly once and 4 weeks later with $20 \mu \mathrm{g}$ LcrE protein combined with Alum adjuvant. As control we used Group 4; mice were immunized with $50 \mu \mathrm{g} \mathrm{p} \Delta \mathrm{RC}$ plasmid intramuscularly two times with 4 -week interval. Two weeks after the last immunization blood was collected in heparinized capillaries from the retro-orbital plexus. All experiments complied with the University of Szeged guidelines for the use of laboratory animals.

\subsubsection{Mouse experiments to study the survival of the recombinant BCG}

We used ApoB100-only LDLR-/- (B6;129S-ApoBtm2SgyLdlrtm1Her/J) mice,16 females per group and their weight ranged from 25-30 $\mathrm{g}$ with the genetic background of a mixture of C57BL/6 and a particular 129 strain from Jackson Laboratories (Bar Harbor, Maine, USA). These mice express full-length ApoB-100 in their LDL particles and have 3-fold higher plasma levels of ApoB100 than those in LDLR-/- mice. The mice were maintained under standard husbandry conditions and were supplied with food and water ad libitum. Intraperitoneal infection of the KO mice was started at 12-14 weeks of age and consisted of $100 \mu \mathrm{l}$ of PBS containing $10^{6}$ colony-forming units (CFU) of either BCGpMV262 or BCGpMV262(icl). Four mice from each group were sacrificed on days 10, 14, 21 and 35 post-infection. The mice were euthanized and their spleens and lungs were excised.

\subsubsection{Culturing of BCG from the lungs and the spleen}

The dissected spleens were homogenized by pressing them through nylon mesh into PBS ( $1 \mathrm{ml})$ containing $0.05 \%$ Tween 20 . The lungs were removed and homogenized mechanically in $1 \mathrm{ml}$ of PBS containing $0.05 \%$ Tween $20.100 \mu \mathrm{l}$ of tissue suspension was used. Ten fold dilutions of these suspensions were plated on Middlebrook 7H10 agar for cultivation of the bacteria. CFUs were determined after 21 days of incubation at $37^{\circ} \mathrm{C}$.

\subsubsection{Western blot}

Cell lysates of E. coli over-expressing ACP, LcrE, Icl and purified control (Chlamydial LcrH), ACP and LcrE proteins and concentrated C. pneumoniae elementary bodies were heated to $95^{\circ} \mathrm{C}$ for 5 min in sample buffer and separated by SDS-10\% PAGE. The separated proteins were blotted onto a polyvinylidene difluoride membrane (SERVA Heidelberg, Germany). The membranes were blocked with PBS containing 5\% skim milk and $0.05 \%$ Tween 20 overnight at $4^{\circ} \mathrm{C}$.

In the case of Icl, membranes were probed with BCGpMV262(icl) immunized and control (BCGpMV262) mouse homogenized lungs (1:10 dilution in 5\% skim milk and $0.05 \%$ Tween 20 containing PBS).

In the case of ACP, membranes were probed with protein-immunized and control (alum only) mouse sera (1:50 dilution in 5\% skim milk and $0.05 \%$ Tween 20 containing PBS). After washings, the filter was incubated with horseradish-peroxidase (HRP)-conjugated antimouse IgG ( $\alpha$-mouse IgG-HRP, Sigma) and the colour was developed using diaminobenzidine tetrahydrochloride (DAB, Sigma) with hydrogen peroxide in $10 \mathrm{mM}$ Tris $\mathrm{pH}$ 7.5.

\subsubsection{ELISA test}

LcrE/ACP-specific antibodies in serial two-fold dilutions of the sera were detected by ELISA, using plates coated with recombinant LcrE/ACP protein (100 ng/well), and HRPconjugated secondary antibody was used for detection. The titres were determined at dilutions demonstrating an optical density (OD) higher than 0.1 .

\subsubsection{Immunization with ACP protein}

Specific-pathogen-free 6-8-week-old, female BALB/c mice were obtained from Charles River Laboratories UK (Kent. England). Mice were maintained under standard husbandry conditions at the animal facility of the department and were given food and water ad libitum. Mice in groups of 5 were immunized subcutaneously at the tail base either with the purified ACP protein diluted in phosphate buffered saline (PBS) at a dose of $20 \mu \mathrm{g}$ mixed with $25 \mu \mathrm{l}$ Alum (Aluminum hydroxide Gel, Sigma) or with Alum only (control) in $150 \mu 1$ volume 3 times at 3 week intervals. Mice were sacrificed 14 days after the last immunization. Blood was collected in heparinized capillaries from the retro-orbital plexus. 


\subsubsection{Challenging the immunized and control mice}

The immunized mice were challenged with $4 \times 10^{5}$ inclusion forming units (IFU) of $C$. pneumoniae (CWL029, ATCC) in $25 \mu 1$ PBS intranasally under pentobarbital sodium anaesthesia. At this time point serum samples were collected in heparinised capillaries by retro-orbital bleeding for testing the antibody production. At 7 days after infection the mice were sacrificed. The lungs were removed and homogenized mechanically in $2 \mathrm{ml}$ of sucrose-phosphate-glutamic acid buffer for cultivation of bacteria.

\subsubsection{Culturing of $C$. pneumoniae from the lungs}

Lung homogenates from individual mice were centrifuged (10 min, $400 \mathrm{~g})$, serial dilutions of the supernatants were inoculated onto HEp-2 cell monolayers and after $48 \mathrm{~h}$ culture cells were fixed with acetone and stained with monoclonal anti-C. pneumoniae antibody (DAKO Ltd. Ely, UK) and FITC-labelled anti-mouse IgG (Sigma). The number of C. pneumoniae inclusions was counted under a UV microscope, and the titre was expressed as IFU/ml.

\subsubsection{Determination of the toxicity of propionate}

BCG bacteria carrying pMV262(icl) or the control plasmid (pMV262) were grown at $37^{\circ} \mathrm{C}$ in Middlebrook $7 \mathrm{H} 9$ (Difco) broth supplemented with OADC. Sodium proprionate $(0.1 \%)$ and/or vitamin $B_{12}(10 \mu \mathrm{g} / \mathrm{ml})$ were used where indicated. Optical density $(600 \mathrm{~nm})$ was measured.

\subsection{Statistical analysis}

Statistical analysis of the data was carried out with GraphPad Prism 5 software, using the Student $t$ test. Differences were considered significant at $\mathrm{P}<0.05$.

\section{Results}

\subsection{ACP as a possible new target molecule for immunization}

\subsubsection{Over-expression of ATB/ACP proteins}

Over-expression of ATB and ACP proteins of M. tuberculosis and C. pneumoniae respectively. ATB is a protein consisting of 248 amino acids. Its calculated molecular mass is 28 $\mathrm{kDa}$; because of the $6 \mathrm{His}$ and FLAG tags, our fusion protein is $4 \mathrm{kDa}$ larger. ACP protein is smaller, it contains 226 amino acids. Its calculated molecular mass is $25 \mathrm{kDa}$; it also contains the 6 His and FLAG tags. Upon heat-induction a strong band appears at the proper position. Since these proteins carry the 6His and FLAG tags, both tags can be exploited during purification. After heat-induction the strong over-expression occurred after 60 minutes in case of both proteins.

\subsubsection{Purification of ACP protein}

The purification was carried out with the TALON CellThru Resin. ACP in the crude extract of heat induced $E$. coli and in the eluted fractions 3 and 4 appears as a strong band around MW $30 \mathrm{kDa}$ position.

\subsubsection{Level of ACP-specific IgG in the sera of the immunized mice}

Mice immunized with ACP protein mixed with Alum adjuvant responded with specific antibody production. ACP-specific IgG was detected by ELISA with a mean titre of 44512.

\subsubsection{Protection against $C$. pneumoniae infection as measured by culturing of $C$. pneumoniae from the lungs}

In order to find out the protective effect of ACP-specific immunity ACP-immunized mice were infected with $C$. pneumoniae. The mean number of recoverable bacteria from the lungs shows $30 \%$ reduction compared to the controls.

\subsubsection{Western blot analysis}

The immunogenicity of the purified protein in mice and the chlamydia-specificity of the produced antibodies were confirmed by Western blot. After immunization with ACP protein, the mouse sera reacted only with a protein at the appropriate position in the concentrated $C$. pneumoniae preparation, with the purified ACP and with ATB protein. This cross reactivity confirmed the epitope similarity between ACP and ATB proteins. These proteins are virulence factors for intracellular bacteria; they are immunogenic and might be eligible components of a 
multi-subunit chlamydia/mycobacterium vaccine. The role of immune response needs further investigation.

\subsection{Modified BCG vaccine may improve the efficacy}

The copy number of the pMV262(icl) in the BCG strain used for infection was 29 . Twenty one days after infection, the spleens from mice that were infected with bacteria that carried the additional $\mathrm{icl}$ genes were three to four times larger than those of the controls.

Bacteria with one copy of $i c l$ (the chromosomal gene) appeared in the spleen earlier and their clearance was faster than that from the lungs. In the spleen, very few bacteria were found 21 days after infection and they had disappeared by the end of the experiment ( 35 days), whereas some bacteria were still detected in the lungs at this time. Bacteria containing the plasmid with the $i c l$ gene survived much longer and their yield was 8-250 times higher. The CFUs were significantly higher in the samples from BCGpMV262(icl)-infected mice than the control $(\mathrm{P}<0.005)$. Lungs from the BCG pMV262(icl)-immunized mice had increased antibody level against Icl.

We determined the toxic effect of propionate produced from the $\beta$-oxidation of oddchain or branched-chain fatty acids. Propionate was less toxic for bacteria carrying icl plasmids in vitro. Although vitamin- $B_{12}$ increased the growth rate of both strains BCG pMV262(icl) multiplied much faster.

\subsection{LcrE experiments}

\subsubsection{Level of LerE specific IgG in the sera of the immunized mice}

All of the mice immunized with LcrE protein mixed with Alum adjuvant, and most of the (7/10) mice immunized with $\mathrm{p} \Delta \mathrm{RCLcrE}$ responded with LcrE specific antibody production. LcrE-specific IgG was detected by ELISA. There was a tendency for higher LcrE-specific IgG level $(\mathrm{p}=0.0625)$ in $\mathrm{p} \Delta \mathrm{RCLcrE}$-primed and LcrE protein boosted (Group 3 ) mice compared to non-primed mice (Group 1).

Significant LcrE-specific IgG1 production was detected in the sera of the LcrE protein immunized mice, however, the sera IgG2a level was low in mice without $\mathrm{p} \Delta \mathrm{RCLcrE}$ priming. Mice immunized with $\mathrm{p} \Delta \mathrm{RCLcrE}$ plasmid only produced relatively higher level of LcrE-specific IgG2a antibody. Boosting the immune response in $\mathrm{p} \Delta \mathrm{RCLcrE}$-primed mice with LcrE protein raised not only the IgG1 but also the IgG2a level. The higher relative IgG2a level indicates a Th1 biased response in the case of DNA immunized mice irrespective of the nature of the booster inoculation.

\subsubsection{Western blot analysis of $\mathbf{p} \Delta \mathrm{RCLcrE-induced} \mathrm{antibodies}$}

The immunogenicity of the eukaryotic expression vector ( $\mathrm{p} \Delta \mathrm{RCLcrE})$ in mice and the LcrE-specificity of the produced antibodies were confirmed by Western blot using PAGEseparated purified LcrE protein as antigen. Identity of purified LcrE protein was previously confirmed by MALDI-TOF mass spectrometry. Sera of mice from Group 3 (immunized $2 \times$ $\mathrm{p} \triangle \mathrm{RCLcrE}$ ) reacted with the band at the position corresponding to the molecular weight of LcrE protein similarly to the sera of LcrE protein immunized-mice of the earlier published experiment.

\subsubsection{Protection against $C$. pneumoniae infection as measured by culturing of $C$. pneumoniae} from the lungs

To find out whether lcrE applied in DNA vaccine can induce protective immune response against $C$. pneumoniae infection, $C$. pneumoniae titres in the lungs of immunized and control mice were compared. The reduction in C. pneumonia burden in the lungs was $37 \%$ in mice immunized with LcrE protein mixed with Alum adjuvant (Group 1); 55\% in $\mathrm{p} \Delta \mathrm{RCLcrE}$ immunized mice (Group 2) and nearly $72 \%$ in $\mathrm{p} \Delta \mathrm{RCLcrE}$-primed and LcrE protein boosted mice (Group 3) when compared to $\mathrm{p} \Delta \mathrm{RC}$-immunized controls (Group 4). This latter comparison showed significant difference with a $\mathrm{P}$ value of 0.01 . 


\section{Discussion}

\subsection{ABC transporter ATPases can be useful components of vaccines}

As shown, we have chosen an important virulence factor of $M$. tuberculosis and its homologous protein from chlamydia, for cloning and expression. The immunogenicity of purified ACP protein and chlamydia-specificity of the produced antibody were proved by Western blot. Low level of protection (albeit not a statistically significant) against $C$. pneumoniae infection was observed by culturing of $C$. pneumoniae from the lungs of ACP immunized mice.

The methods we applied are suitable for cloning, expression and purification of mycobacterial and chlamydial proteins, enabling immunological studies to identify vaccine candidates that would be eligible components of a multi-antigen vaccine which can induce an optimal protective immune response against these infections.

\subsection{Isocitrate lyase encoding plasmids in BCG cause increased survival in mice}

Several vaccines against tuberculosis are currently undergoing clinical trials. Some of them are subunit vaccines. Another approach is the use of recombinant BCG expressing antigens from $M$. tuberculosis. A recombinant $M$. smegmatis was recently shown to induce potent bactericidal immunity against $M$. tuberculosis. The Rv0467 (CAE55284.1) gene codes for Icl in M. tuberculosis H37Rv. In M. bovis BCG, the icl gene (GenBank accession no. CAL70492) differs in only one base and the amino acid composition is identical. The avirulent strain $(M$. tuberculosis $\mathrm{H} 37 \mathrm{Ra}$ ) has the same protein (GenBank accession no. ABQ72194). Sequence search was done using NCBI. Although we cloned icl from H37Rv, because of the identity the extended survival we observed should be due only to the increased copy number. The tricarboxylic acid cycle and the glyoxylate shunt together supply bacteria with both energy and precursors for carbohydrate synthesis from fatty acids. Icl has an important role in the methylcitrate cycle too where the processing of the toxic propionate occurs. Thanks to the suggestion of an anonymous reviewer we determined the growth rate of the bacteria in the presence of propionate in vitro. Bacteria with increased $\mathrm{icl}$ copy multiplied much faster which can contribute to the increased number and survival in mice. Methycitrate cycle and the vitamin $\mathrm{B}_{12}$ - dependent methylmalonyl pathway contribute to mycobacterial pathogenesis by the proper metabolism of propionate during growth on fatty acids in the phagosome. The ,lipid lunch" results in long term survival. Being an enzyme expressed in the later stages of infection by M. tuberculosis, Icl may induce long-term protection if over-expressed from multicopy plasmids during BCG vaccination. In addition, the extended survival of this recombinant BCG itself may increase the efficacy of the vaccine. Western blot shows increased antibody response. It would be interesting to know the protective effect of this recombinant BCG. Because of the lack of biosafety level 3 facilities, we cannot perform these types of experiments. Recent results indicate functions for Icl which extend beyond fat metabolism. A novel metabolic route for carbohydrate metabolism was identified in which Icl is a key enzyme. It was shown that a reduced but constant intracellular ATP level is necessary in the adaptation of $M$. tuberculosis to non-growing survival. Icl deficient nutrient-starved bacilli failed to reduce their intracellular ATP level and died. The mycobacterial cell wall is very rich in lipids: there are around 250 distinct enzymes involved in fatty acid metabolism, five times more than in E. coli. We used ApoB100-only LDLR2/2 mice to gain an insight into the role of a highfat diet on the course of infection, but no significant differences were observed with the different diets in our preliminary experiments (unpublished data). Further studies would be necessary to address this question. It was reported that high levels of cholesterol in the host diet significantly enhanced the M. tuberculosis in the lung and impaired immunity to this pathogen.

\section{3 lcrE-containing DNA immunization}

C. pneumoniae has generated huge attention during the last decade, not only as a respiratory pathogen but because of its possible association with a number of acute and chronic diseases, including atherosclerosis, Alzheimer's disease and multiple sclerosis. The true linkage and causality of $C$. pneumoniae infection in the development of chronic manifestations is poorly understood. Since antibiotics cannot fully inhibit chlamydial growth and because of the incomplete protection induced by natural infection, the development of an effective vaccine 
would be desirable to control the infections caused by this highly prevalent pathogen. With recent advances in chlamydial genomics and proteomics, a preference has developed for subunit vaccines. The multi-subunit approach to chlamydia vaccine will likely induce an effective longlasting immunity. Structural proteins of TTSS are expected to be surface exposed and required for infection of host cells, thus, these are attractive vaccine candidates to investigate. Protective effect of LcrE-specific immunity against $C$. pneumoniae infection in animal models has been studied. The results of our published experiments are in agreement with previous reports that LcrE protein is an antigen with considerable potential as a vaccine subunit and in this study we confirmed that this antigen has protective effect formulated as a DNA vaccine, too. In our present study we show that we cloned $l c r E$ gene into a eukaryotic expression vector, and $l c r E$-DNA immunization induced protective response against $C$. pneumoniae infection similar to that evoked by a single LcrE protein immunization. Moreover, in a prime-boost regime, i.e. lcrE DNA/LcrE protein vaccination, the protective effect reached $72 \%$ in respect of lung bacterial burden. This level of protection is significant and comparable to that achieved by multiple subcutaneous injection of LcrE protein mixed with Alum adjuvant as published earlier. Our findings are that $\mathrm{p} \Delta \mathrm{RCLcrE}$ immunization induces an immune response shifted towards Th1 type pathway characterized by increased IgG2a production, and immunization with LcrE protein which generates an IgG1dominated Th2 type response did not alter the DNA priming-polarized Th1 type response in DNA-primed and protein-boosted mice. Further experiments with improved immunization regimens or modified plasmid vectors are planned for inducing an increased level of antibody and/or cellular response potentially leading to better protection.

\section{The following of our results are considered novel}

- $\quad \mathrm{ABC}$ transporter ATPases can be useful components of vaccines, we showed that chlamydial ATPase induces protective immunity in mice

- Isocitrate lyase encoding plasmids in BCG cause increased survival in mice, this recombinant $\mathrm{BCG}$ can be a more effective vaccine

- $\quad l c r E$-containing DNA immunization given as a priming and followed by a protein booster significantly reduced the number of viable bacteria in the lungs of mice after challenge with $C$. pneumoniae

\section{Summary}

Tuberculosis (TB) has troubled humankind throughout history. It has been a leading cause of death, and still is in low- and middle-income countries. TB is a classic example of airborne infection. The causative agent, M. tuberculosis, is a very successful pathogen. One third of the human population is infected with this bacterium. WHO declared TB a public health emergency in 1993. The available vaccine, bacillus Calmette-Guérin (BCG) has variable (generally low) efficacy in different parts of the world and no new drugs were introduced in the last 40 years. Multidrug- and extensively drug-resistant (MDR and XDR, respectively) strains have developed.

C. pneumoniae is an obligate intracellular human pathogen, which causes respiratory tract infections, i.e. pneumonia, bronchitis, pharingitis and sinusitis. Seroepidemiological studies show that $C$. pneumoniae is widespread and nearly everybody becomes infected with it during his life. C. pneumoniae can also cause chronic infections, which might lead to atherosclerosis and heart disease. It is suspected to have a role in the pathogenesis of Alzheimer disease and multiple sclerosis. These are the reasons why it is important to develop vaccine against $C$. pneumoniae infection. Better vaccines and new therapeutic drugs could be a successful breakthrough against intracellular bacteria. 
M. tuberculosis ABC transporter ATPase (Rv0986) plays a role in mycobacterial virulence by inhibiting phagosome-lysosome fusion. Thus, it could be a potential vaccine candidate. $C$. pneumoniae possesses a protein named $\mathrm{CpB} 0255$, which is homologous with the mycobacterial Rv0986. We cloned, over-expressed and purified these bacterial ABC transporter ATPase proteins to study their biological properties. The immunogenicity and protective effect of recombinant chlamydial ATPase protein combined with Alum adjuvant were investigated in mice. The immunization resulted in the reduction of the number of viable C. pneumoniae in the lungs after challenge. Our results confirm that chlamydial ATPase induces protective immunity in mice, and the produced antibody also recognized mycobacterial ATPase protein, which justifies the epitope homology between these proteins.

We studied the role of isocitrate lyase in the interaction between $M$. bovis $\mathrm{BCG}$ and mice. ApoB100-only LDLR-/- (B6;129S-ApoBtm2SgyLdlrtm1Her/J) mice were inoculated with $M$. bovis BCG harbouring plasmids carrying the gene for isocitrate lyase. The presence of $\sim 29$ times more copies of this gene resulted in a higher bacterial yield in the spleens and lungs of the infected mice. The spleen was 3-4 times heavier, and in the spleen the bacteria survived over 10 days longer than did the bacteria with the control plasmid. Propionate was less toxic for bacteria carrying icl plasmids in vitro. This recombinant BCG can be a possible vaccine candidate.

C. pneumoniae possess TTSS, which allows them to secrete effector molecules into the inclusion membrane and the host cell cytosol. Low calcium response protein $\mathrm{E}$ (LcrE) is a part of TTSS. The gene of LcrE in a 6His-tagged form was cloned from C. pneumoniae CWL029, expressed and purified from E. coli using the HIS-select TALON CellThru Resin, this gene was also cloned into a eukaryotic expression vector $(\mathrm{p} \triangle \mathrm{RC})$. One group of $\mathrm{BALB} / \mathrm{c}$ mice received an intramuscular $\mathrm{p} \triangle \mathrm{RC}$ inoculation then the mice were immunized with purified LcrE protein; the second group of mice was immunized two times with the recombinant plasmid ( $\mathrm{p} \Delta \mathrm{RCLcrE}$ ), and the third group was primed with $\mathrm{p} \Delta \mathrm{RCLcrE}$ inoculation then boosted with LcrE protein. LcrEspecific antibody response was induced by DNA immunization with a shift towards Th1 isotype pattern compared to protein-immunization, this shifting pattern was observed in plasmid primed then protein-boosted animals. DNA immunization given as a priming and followed by a protein booster significantly reduced the number of viable bacteria in the lungs after challenge with $C$. pneumoniae. These results confirm that immunization with $\mathrm{p} \Delta \mathrm{RCLcrE}$ can be an effective part of a vaccination schedule against $C$. pneumoniae

\section{Acknowledgements}

I would like to express my great appreciation to everyone who has contributed along the way during my Ph.D. studentship years.

I would especially like to mention the following people:

András Miczák, my supervisor, and Ildikó Faludi for sharing their knowledge. This thesis would not have been possible without their help and support.

Professor Yvette Mándi, head of the Doctoral School of Interdisciplinary Medicine, for accepting me as Ph.D. student, former head of the Department of Medical Microbiology and Immunobiology and Katalin Burián the present head, for providing working facilities.

Mrs Lévai for her excellent technical assistance and advice.

All my fellow Ph.D. students for creating a great atmosphere at work and nice times during coffee breaks.

Past and present colleagues and staff members at the Department of Medical Microbiology and Immunobiology, for welcoming me to the lab and for all their help. Special thanks to Professor József Molnár, Valéria Endrész and Gabriella Spengler for kindness, support and help during these years.

All my old Friends, for always being there and for having no idea what I do at work.

My Family and my Love who have always supported, encouraged and believed in me.

This dissertation is dedicated to my Mother. 
This work was supported by Hungarian Ministry of National Resources; by TÁMOP-4.2.2/B10/1-2010-0012 project: "Broadening the knowledge base and supporting the long term professional sustainability of the Research University Center of Excellence at the University of Szeged by ensuring the rising generation of excellent scientists." The project is supported by the European Union and co-financed by the European Social Fund.

\section{Publications related to the thesis}

I. Szabó AM, Endrész V, Somogyvári F, Miczák A, Faludi I. Isocitrate lyase encoding plasmids in BCG cause increased survival in ApoB100-only LDLR-/- mice. Mol Biol Rep. 2013; 40(8):4721-5.

Impact factor: 2,929

II. Szabó AM, Sipák Z, Miczák A, Faludi I. ABC transporter ATPase of Chlamydophila pneumoniae as a potential vaccine candidate. Acta Microbiol Immunol Hung. 2013; 60(1):11-20.

Impact factor: $\mathbf{0 , 7 8 7}$

III. Faludi I, Szabó AM. Vaccination with DNA vector expressing chlamydial low calcium response protein E (LcrE) against Chlamydophila pneumoniae infection. Acta Microbiol Immunol Hung. 2011; 58(2):123-34. Impact factor: 0,787

IV. Faludi I, Szabó AM, Burián K, Endrész V, Miczák A. Recombinant Mycobacterium smegmatis vaccine candidates. Acta Microbiol Immunol Hung. 2011; 58(1):13-22.

Impact factor: $\mathbf{0 , 7 8 7}$

\section{Publications not directly related to the thesis}

I. Varga GZ, Szabó AM, Kerényi M, Molnár J. Interference in Quorum sensing signal transmission amongst microbial species. Acta Microbiol Immunol Hung. 2012; 9(4):477486.

Impact factor : $\mathbf{0 . 7 8 7}$

II. Varga GZ, Szabó AM, Schelz Zs, Szegedi E, Amaral L, Molnár J. Quorum sensing inhibition by phenothiazines and related compounds. Lett Drug Des Discov. 2011; 8(2):133-137.

Impact factor: 0.668

III. Szabó AM, Varga GZ, Hohmann J, Schelz Zs, Szegedi E, Amaral L, Molnár J. Inhibition of Quorum Sensing by Essential Oils. Phytother Res. 2010;24:782-86.

Impact factor: 1.878

IV. Faludi I, Csanádi A, Szabó AM, Burián K, Endrész V, Miczák A. Production and purification of low calcium response protein $\mathrm{H}$ of Chlamydophila pneumoniae. Acta Microbiol Immunol Hung. 2009; 56(4):389-97.

\section{Conference abstracts:}

2012:

Spengler G, Rodrigues L, Martins M, McCusker M, Santos Costa S, Ntokou E, Martins A, Szabó AM, Horváth Á, Varga GZ, Fanning S, Molnár J, Amaral L. Stress response and resistance of Salmonella enterica serotype Enteritidis to the efflux pump inhibitor neuroleptic drug thioridazine; $22^{\text {nd }}$ ECCMID, 31 March - 3 April, 2012 London, United Kingdom

2011:

Szabó AM, Faludi I, Miczák A. BCG carrying ICL gene on plasmid shows increased survival in mice; $21^{\text {st }}$ ECCMID/27 $7^{\text {th }}$ ICC, 7-10 May, 2011 Milan, Italy 
Faludi I, Szabó AM, Burián K, Mosolygó T, Miczák A, Endrész V. Low calcium response protein $\mathrm{E}$ (LcrE) vaccination is effective in DNA priming/protein booster form against Chlamydophila pneumoniae infection in BALB/c mice; 16th International Congress of the Hungarian Society for Microbiology, 20-22 July, 2011 Budapest, Hungary

2010:

Szabó AM, Faludi I, Miczák A. Meropenem sensitivity of mycobacteria; International Congress of the Hungarian Society for Microbiology, 2010 Keszthely, Hungary

Faludi I, Szabó AM, Burián K, Miczák A, Endrész V. Low calcium response protein E, H and $\mathrm{ABC}$ transporter ATPase of Chlamydophila pneumoniae; 6. PhD Symposium, HÖRSAALZENTRUM OF THE MEDICAL UNIVERSITY OF VIENNA GENERAL HOSPITAL OF VIENNA 16-17 June, 2010 Vienna, Austria

Varga GZ, Szabó AM, Schelz ZS, Szegedi E, Molnár J. Quorum Sensing Inhibition by Phenothiazines and Essential Oils; 6. PhD Symposium, HÖRSAALZENTRUM OF THE MEDICAL UNIVERSITY OF VIENNA GENERAL HOSPITAL OF VIENNA 16-17 June, 2010 Vienna, Austria 